\title{
Molecular and immune response characterizations of IL-6 in large yellow croaker (Larimichthys crocea)
}

\author{
Qian Zhu ${ }^{\dagger}$, Chan $\mathrm{Li}^{\dagger}$, Zhen-Xing Yu, Peng-Fei Zou, Qing-Xiang Meng, Cui-Luan \\ Yao*
}

Fisheries College/ Fujian Provincial Key Laboratory of Marine Fishery Resources and Ecoenvironment, Jimei University, Xiamen 361021, PR China

\section{ABSTRACT}

Interleukin-6 (IL-6) is a multifunctional inflammatory cytokine which exists in multiple tissues and cell lines. In the present study, the full-length cDNA and the genomic sequence of IL-6 (LcIL-6) were cloned from large yellow croaker, Larimichthys crocea. The full-length cDNA of $L c$ IL-6 was 1066 base pairs (bp), containing an open reading frame (ORF) of $678 \mathrm{bp}$ encoding for 225 amino acids, a 5' untranslated region (UTR) of $71 \mathrm{bp}$ and a 3' UTR of $317 \mathrm{bp}$. The predicted $L c \mathrm{IL}-6$ protein included a 24 amino acids (aa) signal peptide and a conserved IL-6 domain. However, the polypeptide sequence identities between $L c \mathrm{IL}-6$ and its counterparts in mammals and other fish are from $12 \%$ to $45 \%$. The genome sequence of $L c \mathrm{IL}-6$ gene was composed of $2126 \mathrm{bp}$, including five exons and four introns. Phylogenetic analysis revealed that $L c$ IL-6 showed a close relationship with the IL-6 from other bony fish. Quantitative real-time PCR (qRT-PCR) analysis revealed that $L c$ IL6 mRNA was expressed in most examined tissues, with the most predominant expression in stomach,

\footnotetext{
${ }^{\dagger}$ Both authors have the same contribution.

* Corresponding author: C.L. Yao, Email: clyao@jmu.edu.cn
} 
followed by blood and very weak expression in other tissues. The expression levels of $L c \mathrm{IL}-6$ after challenged with LPS, poly I:C and Vibrio parahaemolyticus were investigated in spleen, headkidney and liver. $L c$ IL-6 transcripts were induced significantly after immune challenge, with the peak-value of 33.5 times as much as the control in the head-kidney at $3 \mathrm{~h}$ after LPS injection ( $p<$ 0.05). Overexpression of $L c \mathrm{IL}-6$ enhanced tumor necrosis factor (TNF)- $\alpha$ transcripts significantly $(p<0.05)$ in $L$. crocea kidney (LCK) cells. Additionally, recombinant $L c$ IL-6 mature peptide was obtained in the supernatant of Escherichia coli BL21 (DE3). The purified recombinant LcIL-6 fusion protein was also demonstrated to improve the transcriptional expression levels of TNF- $\alpha$ significantly in LCK cells $(p<0.05)$. However, no significant changes of Mx (myxovirus resistant protein), IL-1 $\beta$, janus kinase (JAK)2, signal transducers and activators of transcription (STAT) 3 and STAT5 in LCK cells was detected after $L c$ IL-6 overexpression or recombinant $L c$ IL-6 protein stimulation. Our results indicated that $L c$ IL-6 might be important in large yellow croaker immune response and improve the inflammatory response by through activation TNF- $\alpha$ expression.

Key words: Interleukin-6; gene structure; large yellow croaker; immune challenge; overexpression; recombinant expression; inflammatory response

(This research was supported by NSFC (31101882; 41276178) and 973 Program (2011CB111604) to C.L.Y) 